\title{
Mast cell activation disorder masquerading as a nervous breakdown
}

\author{
Anh P. Nguyen*1, Suzanne S. Teuber ${ }^{1,2,3}$ \\ ${ }^{1}$ Department of Internal Medicine, University of California Davis Medical Center, Sacramento, United States \\ ${ }^{2}$ Division of Rheumatology, Allergy and Clinical Immunology, University of California Davis Medical Center, Sacramento, United \\ States \\ ${ }^{3}$ Veterans Affairs Northern California Healthcare System, Mather, United States
}

Received: May 1, 2017

DOI: $10.5430 /$ crim.v4n3p51
Accepted: July 6, 2017

Online Published: July 18, 2017

URL: https://doi.org/10.5430/crim.v4n3p51

\begin{abstract}
While mast cells have been implicated in human disease since the early 1900s, mast cell activation syndrome (MCAS) was only recently discovered and recognized as a disorder caused by aberrant release of mast cell mediator enzymes. These mediators trigger a wide variety of symptoms ranging from headache, pruritis and gastrointestinal discomfort to life-threatening anaphylaxis. MCAS is frequently unrecognized and misdiagnosed because symptoms associated with MCAS are often present in other medical conditions and are highly variable among patients. We describe a case of a 62-year-old male who presented to the emergency department (ED) with three days of recurrent flushing, diarrhea, mild edema of his hands bilaterally, and severe emotional lability with recurrent crying. In the ED, he was misdiagnosed with severe anxiety and directly sent to a mental health clinic for assistance. However, he was previously seen in allergy clinic for suspected MCAS. Fortuitously standing labs including plasma histamine, tryptase, and urine studies that were ordered in allergy clinic were drawn concurrently with the initial ED workup. He was found to have elevated histamine level during his ED episode and treatment for MCAS was initiated. In subsequent visits, his symptoms, except for episodes of "brain fog" and emotional lability, improved significantly on a combination of antihistamines, oral cromolyn sodium, and montelukast. Additionally every other day prednisone has substantially cleared the neurocognitive symptoms. This case highlights the difficulty of MCAS diagnosis and the importance of assessing for MCAS, especially in patients who have multisystem complaints.
\end{abstract}

Key Words: Mast cell, Mast cell activation disorder, Flushing, Diarrhea, Psychiatric

\section{INTRODUCTION}

Mast cells (MCs) are immune system cells derived from myeloid stem cells and undergo final differentiation in peripheral tissue. They serve both the innate and adaptive immune systems in various body tissues, particularly skin, gastrointestinal and airway tracts. Mast cells mediators, including histamine, are responsible for various allergic inflammatory symptoms. ${ }^{[1,2]}$
MCs were first implicated in human disease when Dr. Unna, a dermatologist, found presence of MCs in urticarial pigmentosa - pigmented maculopapular lesions. ${ }^{[3]}$ Nearly six decades later, a pathologist, Dr. John Ellis, discovered MC involvement in organ systems in a 1-year-old child who died of cachexia, leading to the first description of systemic mastocytosis (SM). ${ }^{[4]}$ In 1957, Efrati et al. described the first clear case of mast cell leukemia. ${ }^{[5]}$ Later on, it was found

*Correspondence: Anh P. Nguyen; Email: aphnguyen@ucdavis.edu; Address: Department of Internal Medicine, University of California Davis Medical Center, Sacramento, United States.

Published by Sciedu Press 
and accepted that among the MC mediators, tryptase, a serine proteinase enzyme, is a sensitive and specific marker for mast cell activation in mastocytosis. ${ }^{[6]}$ It was thought that mast cell disease exist in two forms, cutaneous and systemic, and are caused by abnormal mast cell proliferation. However, since its discovery there have been reported cases of MC-mediated symptoms with normal or only slightly elevated tryptase levels and without cutaneous evidence of mast cell disease, and not meeting the criteria for systemic mastocytosis on bone marrow evaluation. ${ }^{[6,7]}$ In 2010, Akin et al. proposed new diagnostic criteria for mast cell activation disorders (MCAD) which includes clonal cutaneous and systemic mastocytosis as well as clonal and non-clonal mast cell activation syndromes (MCAS). ${ }^{[8,9]}$

Symptoms of MCAS can occur at any age. ${ }^{[9-11]}$ Common clinical features of MCAS include flushing, abdominal cramping, diarrhea, hypotensive syncope, wheezing and pruritus. Rarely, patients also present with cognitive distur- bances as well as frank psychosis. ${ }^{[10,11]}$ Due to the clinical heterogenity of MCAS and low awareness of MCAS, the diagnosis is often missed. In this report, we present a case of MCAS misdiagnosed as anxiety disorder.

\section{CASE PRESEntation}

A 62-year-old male without prior psychiatric disorder presented to the emergency department (ED) with chief complaint of "foggy brain", emotional lability, severe diarrhea and flushing that started three days prior. He was very tearful and felt that he was not functional. He was thought to have severe anxiety. He was discharged from the ED and sent to the mental health urgent crisis clinic for evaluation without further medical workup, but some labs that were already on order in the electronic medical record by outpatient allergist were drawn. The mental health clinic suspected underlying medical illness and referred the patient back to his primary care doctor (see Figure 1).

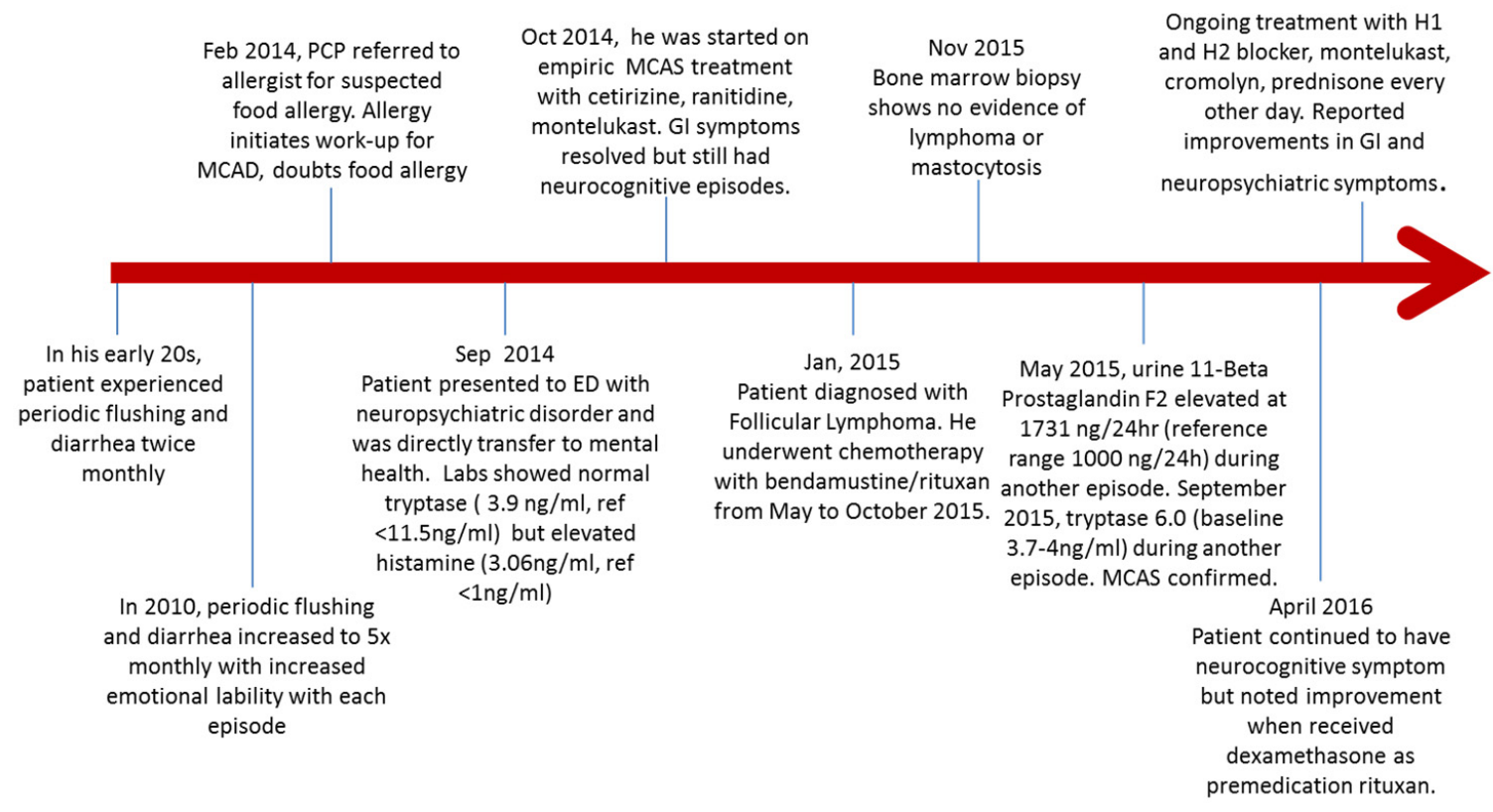

Figure 1. Patient's timeline

He had in fact recently been seen in the Allergy Clinic for suspected "tree nut allergy" about six months prior to the ED visit. At that visit, the patient reported that he had years of intermittent diarrhea and food-poison like reactions, especially after eating mixed nuts, since he was in his early 20s. Flushing of the face and sometimes flushing of his arms often precede the diarrhea. The flushing and diarrhea symptoms used to occur once to twice a year since his early 20s; however, in the last five years, he had these episodes more frequently and he would also get "brain fog" and become very emotional with each episode. Patient noted that the cognitive dysfunction affected mostly his short term memory. He works as a casino dealer, and during his last episode, he had trouble doing calculations that are normally easy for him, resulting in monetary loss at work.

Past medical history was negative for allergic rhinitis, urticaria, and insect sting allergy. Physical exam was notable for lack of lymphadenopathy and hepatosplenomegaly. Skin exam revealed several brown papules and nodules on the lateral right thigh and foot; however, Darier's sign was negative. 
His allergist suspected at that visit that the patient did not have food allergy, but may have a mast cell disorder. Orders were placed for serum tryptase, plasma histamine, 24 hour urine for $\mathrm{N}$-methylhistamine and prostaglandin metabolites. The patient did not get these labs at baseline, but when he presented to the ED, the tryptase and plasma histamine were drawn. ED labs revealed elevated plasma histamine $3.06 \mathrm{ng} / \mathrm{ml}$ (reference $1.00 \mathrm{ng} / \mathrm{ml}$ ). Concurrent tryptase level was normal. The urine tests were not done.

Infectious workup for the diarrhea including stool bacterial cultures and ova and parasites were negative. Serology for celiac disease was also negative. Due to the flushing episodes, he was further ruled out for carcinoid tumor and pheochromocytoma. Specific IgE to nuts (peanut, almond, Brazil nut, hazelnut, cashew, walnut, pecan, and pistachio) were also negative. Subsequent VIP, gastrin, chromogranin A were all normal. IL-6 was normal, ESR was 3, HS-CRP was 1.5 which are contrary to an auto-inflammatory syndrome. A dermatologist biopsied one of the suspicious skin lesions and it was found to be consistent with dermatofibrosis and not cutaneous mastocytosis.

The patient was empirically started on cromolyn sodium, antihistamine (H1, H2 blocker) and montelukast (LTRA antagonist) with reported marked improvement in symptoms. Episodes of flushing and diarrhea occurred much less frequently and were shorter in duration. However he still had neuropsychiatric symptom of mood liability and "brain fog" that were disabling to him. It was not until nine months after his ED visit that another lab test, urine 11-Beta Prostaglandin F2, was correctly obtained during one of his episodes. It was elevated at $1,731 \mathrm{ng} / 24 \mathrm{~h}$ (reference range $1,000 \mathrm{ng} / 24 \mathrm{~h}$ ) and was in normal range when asymptomatic. The unique constellation of symptoms, elevated histamine and urine 11$\mathrm{B}$ prostaglandin $\mathrm{F} 2$ during episodes and normal in-between, repeatedly normal tryptase, in combination with patient's symptom improvement on therapy confirmed the diagnosis of MCAS.

A few months after he was started on empiric MCAS treatment, he lost $30 \mathrm{lbs}$ which he attributed to a change of diet by avoiding all food he thought might be triggering flushing and diarrhea. However, on exam, enlarged left inguinal nodes were palpated leading to further malignancy workup. PET scan revealed hypermetabolism in a left external iliac and several left inguinal nodes suspicious for possible neoplasia. Subsequent biopsy of the lymph node confirmed Grade 1 follicular lymphoma (FL).

He was started on bendamustine/rituximab. PET scan three months later revealed resolution of previous hyper- metabolism in the left external iliac and left inguinal nodes and no abnormal FGD uptake. Subsequent bone marrow biopsy showed no evidence of lymphoma, normocellular marrow, nor evidence of systemic mastocytosis. The bone marrow biopsy's immunohistochemical stain showed evidence of increased CD2 on T-cells, but no increased CD25, CD117 (c-KIT) nor Tryptase activities.

He continued to have episodic diarrhea, "foggy" feeling, and swelling of hands and legs, though much improved while on medications. Maintenance rituximab (q2monthly) was started in part as it was felt to possibly help with symptoms of MCAS as well as FL. He noted he felt "great" for a few weeks after every treatment.

One year after starting the combination of antihistamines, cromolyn sodium and montelukast, the patient reported that he had "180 degree" improvement in his GI symptoms. However, he was still getting "foggy brain" as well as arthralgia and fluid retention. He was tried on aspirin therapy (tried initially on very low dose and uptitrated) but did not tolerate the bruising and did not feel it helped. Test dosing with the dexamethasone that he was given as a premedication with Rituximab showed that it was the most likely the episodic corticosteroid that would result in several weeks of great improvement in all symptoms. Since the neurocognitive symptoms were resistant to current MCAS therapy, he was started on prednisone $8 \mathrm{mg}$ every other day for symptom control after careful discussion of the risks and benefits. Patient reported significant improvement in all symptoms, especially his neuropsychiatric symptoms after starting on prednisone. Rituximab was stopped in due course and he remains with no evidence of lymphoma. He is currently continued on every other day prednisone, in addition to the antihistamines, cromolyn sodium and montelukast.

\section{DiscuSSION}

Mast cell activation causes degranulation of mediators, including histamine, heparin, and various proteases, as well as synthesis of arachidonic acid metabolites and cytokines ${ }^{[7,12]}$ (see Figure 2).

Histamine acts on $\mathrm{H} 1$ receptors which cause contraction of bronchial and GI smooth muscle. It also stimulates parietal cells via $\mathrm{H} 2$ receptors causing increased gastric acid production Prostaglandin D2, leukotriene C4, tryptase, and inflammatory cytokines cause wide inflammatory response. ${ }^{[7,12]}$ Together these mediators cause the common presenting clinical features of MCAS including flushing, abdominal cramping, diarrhea, hypotension, wheezing, and pruritus. Although rare, some patients can present with minor neurocognitive disturbances as well as frank psychotic behaviors. ${ }^{[8,10]}$ 


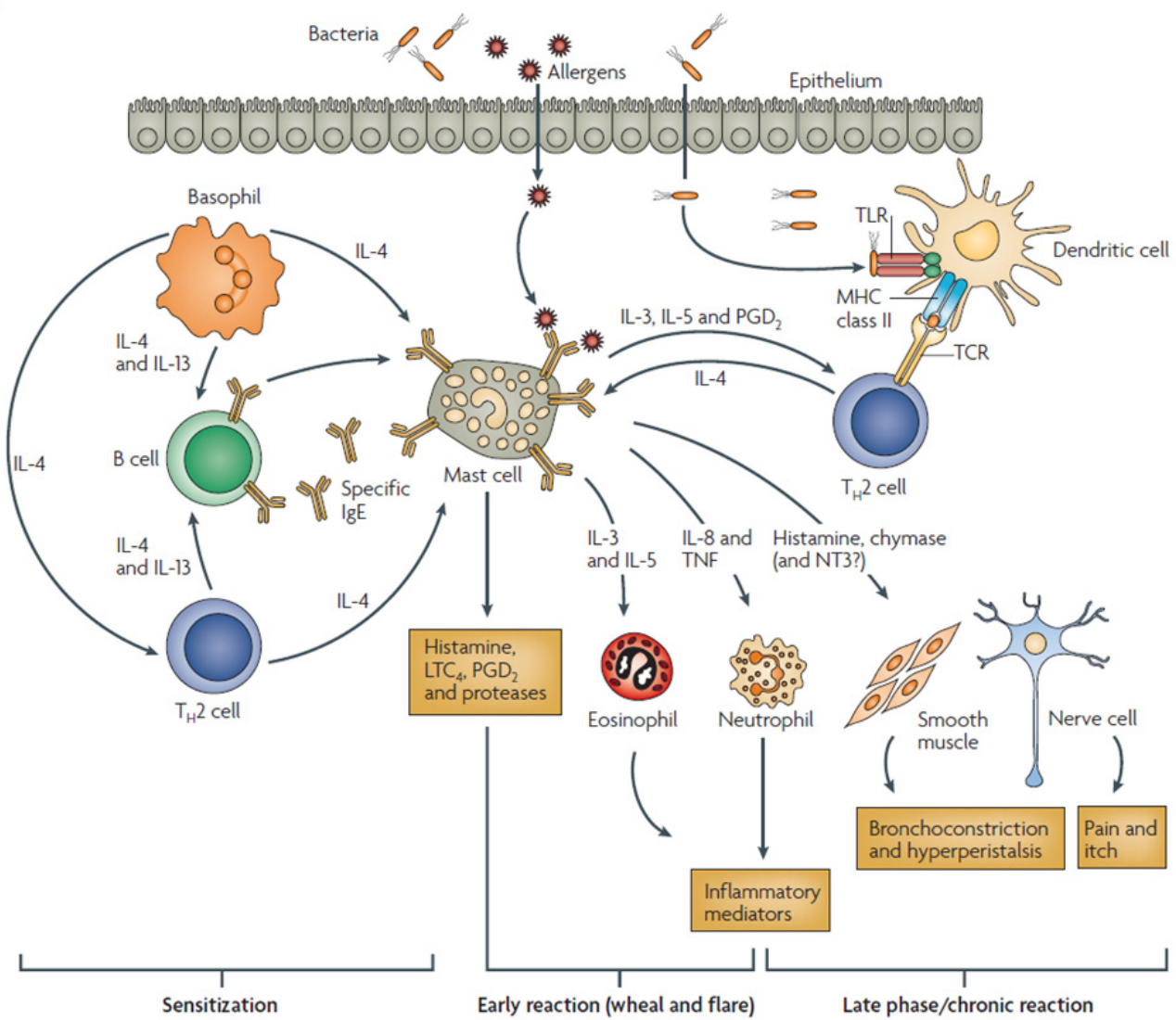

Figure 2. Mast cell activation pathway (Figure taken from Stephan ${ }^{[2]}$ )

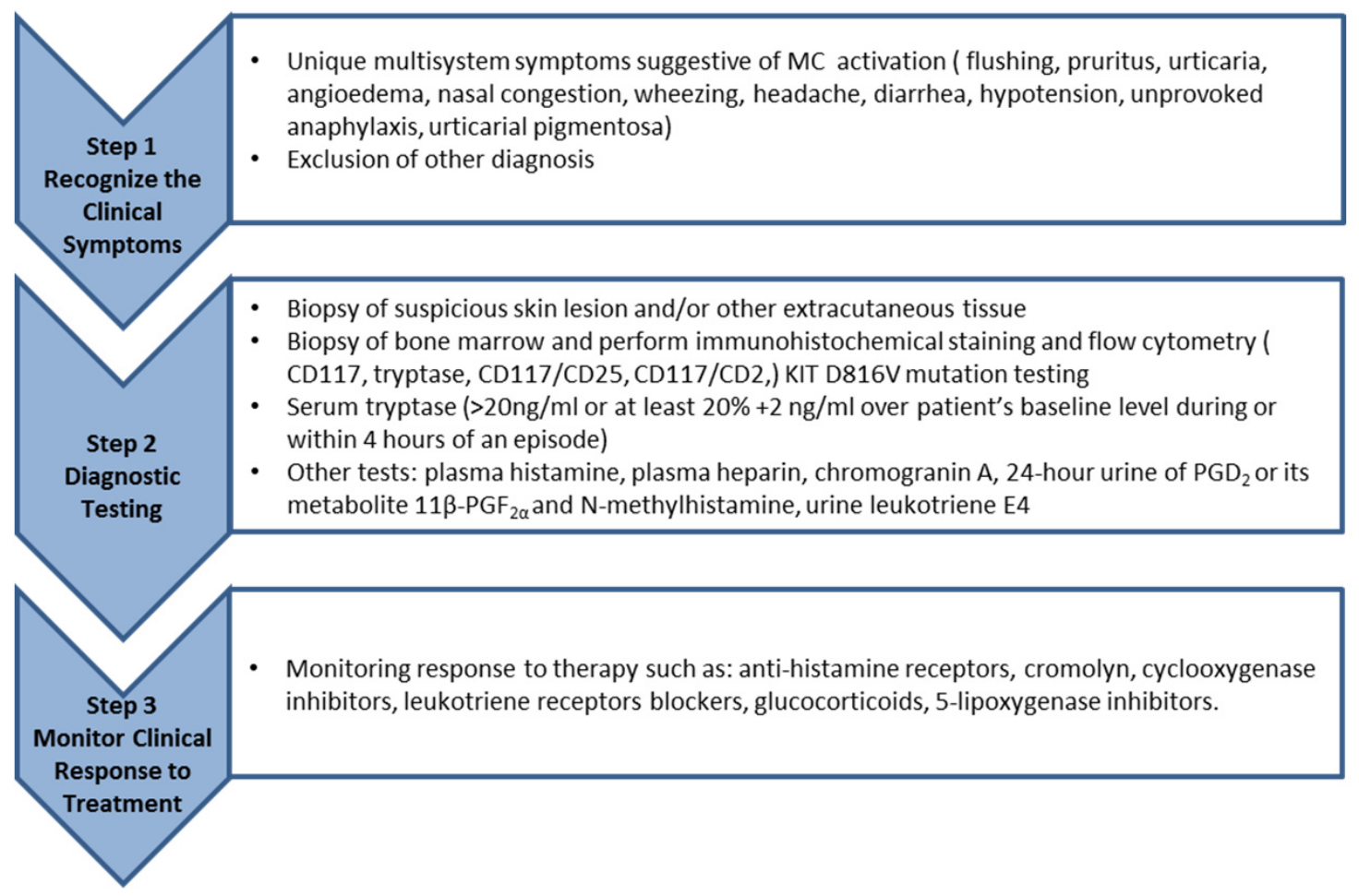

Figure 3. Algorithmic approach of diagnosing MCAD (Figure adapt from Afrin et al. ${ }^{[9,13]}$ ) 
Prior to 2007, MCAS was not a recognized diagnosis. However it became more apparent with further research and case reports that systemic mastocytosis and cutaneous mastocytosis are rarer forms of MCAD that are cause by abnormal mast cell proliferation due to c-kit mutation of tyrosine kinase. ${ }^{[8-13]} \mathrm{MCAS}$, in contrast, can be a result of another MC disorder, such as mastocytosis (primary MCAS) or IgE-dependent disease due to an allergy (secondary MCAS) or idiopathic activation of MCs mediators. In 2010, a conference was organized to propose a global classification of

\begin{tabular}{|c|c|}
\hline Criteria to define mast cell activation syndrome & WHO criteria to define systemic mastocytosis \\
\hline Major criteria & Major criterion \\
\hline $\begin{array}{l}\text { 1. Multifocal or disseminated dense infiltrates of mast cells in } \\
\text { bone marrow biopsies and/or in sections of other extracutaneous }\end{array}$ & \\
\hline $\begin{array}{l}\text { organ(s) (e.g., gastrointestinal tract biopsies; CD117-, tryptase- } \\
\text { and CD25-stained). }\end{array}$ & $\begin{array}{l}\text { Multifocal dense infiltrates of mast cells ( }>15 \text { mast cells in } \\
\text { aggregates) in bone marrow biopsies and/or in sections of other }\end{array}$ \\
\hline $\begin{array}{l}\text { 2. Unique constellation of clinical complaints as a result of a } \\
\text { pathologically increased mast cell activity (mast cell mediator } \\
\text { release syndrome). }\end{array}$ & extracutaneous organ(s) (CD117-, tryptase- and CD25-stained). \\
\hline Minor criteria & Minor criteria \\
\hline $\begin{array}{l}\text { 1. Mast cells in bone marrow or other extracutaneous organ(s) } \\
\text { show an abnormal morphology }(>25 \%) \text { in bone marrow smears } \\
\text { or in histologies. }\end{array}$ & $\begin{array}{l}\text { 1. Mast cells in bone marrow or other extracutaneous organ(s) } \\
\text { show an abnormal morphology }(>25 \%) \text { in bone marrow smears } \\
\text { or in histologies. }\end{array}$ \\
\hline 2. Mast cells in bone marrow express CD2 and/or CD25 & 2. Mast cells in bone marrow express CD2 and/or CD25. \\
\hline $\begin{array}{l}\text { 3. Detection of genetic changes in mast cells from blood, bone } \\
\text { marrow or extracutaneous organs for which an impact on the } \\
\text { state of activity of affected mast cells in terms of an increased } \\
\text { activity has been proved. }\end{array}$ & $\begin{array}{l}\text { 3. c-kit mutation in tyrosine kinase at codon } 816 \text { in mast cells in } \\
\text { extracutaneous organ(s). }\end{array}$ \\
\hline $\begin{array}{l}\text { 4. Evidence of a pathologically increased release of mast cell } \\
\text { mediators by determination of the content of }\end{array}$ & \\
\hline - tryptase in blood & 4. Serum total tryptase $>20 \mathrm{ng} / \mathrm{ml}$ (does not apply in patients \\
\hline - N-methylhistamine in urine & who have associated hematologic non-mast-cell lineage \\
\hline - heparin in blood & \\
\hline - chromogranin A in blood & \\
\hline
\end{tabular}

Note. MCAS is diagnosed if two major criterions, or second criteria and one minor criterion are met. SM diagnosis is made when one major criterion and one minor or $\geq$ three

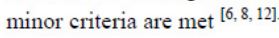

Figure 4. Mast cell activation disease criteria (Figure taken from Moldering et al., ${ }^{[6]}$ published in Biomed Central)

At that time, further testing can determine whether patient has monoclonal MC (primary MCAS), allergy or another underlying disease causing MCA (secondary MCAS), or idiopathic (non-clonal) MCAS. ${ }^{[14]}$ Primary MCAS includes those who meet MCA criteria and has MC (mono) clonality (CD25 + and/or KIT D816V). Primary MCAS can be further divided into mastocytosis (systemic mastocytosis and cutaneous mastocytosis) and (Mono)clonal MCAS ${ }^{[9]}$ (WHO criteria, see Figure 4). Some argue that distinguishing between monoclonal MCAS and "non-clonal" MCAS is of little significant as the clonality is seen rarely in MCAS and only in small number of mastocytosis patients and therapeutic
MCAS and the following criteria is now used to diagnose systemic mast cell activation (MCA): 1) typical clinical manifestations, 2) increase in tryptase (at least $20 \%+2 \mathrm{ng} / \mathrm{ml}$ over patient's baseline level during or within 4 hours of an episode), elevated histamine/histamine metabolites and 24hour urine PGD2 or its metabolite $11 \beta-\mathrm{PGF}_{2 \alpha}, 3$ ) improvement of clinical symptoms to anti-mediator pharmacological agent, especially histamine receptor blocker. If patient meet all three criteria, MCA is diagnosed ${ }^{[9,13]}$ (see Figures 3 and 4).

\section{WHO criteria to define systemic mastocytosis}

aggregates) in bone marrow biopsies and/or in sections of other extracutaneous organ(s) (CD117-, tryptase- and CD25-stained).

\section{Iinor criteria}

. Mast cells in bone marrow or other extracutaneous organ(s) or in histologies.

2. Mast cells in bone marrow express CD2 and/or CD25.

3. c-kit mutation in tyrosine kinase at codon 816 in mast cells in

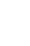


molecular testing to look for KIT mutation D816V. His IgE panel was also negative which suggests against IgE-driven pathology. As we did not perform chromosomal analysis to look for KIT mutation D816V, we cannot completely rule out primary MCAS but with the current findings our patient likely has idiopathic MCAS.

MCAS management mainly target mast cell mediated symptoms. As MCAS is not usually proliferative, cytotoxic agents are rarely utilized. Aspirin, corticosteroids, and rarely immunomodulatory drugs are utilized to inhibit mediator production. Mast cell stabilization agents such as cromolyn, and sometimes tyrosine kinas inhibitor and/or omalizumab can help block MCs mediators release. Additionally, histamine $\mathrm{H} 1 / \mathrm{H} 2$ blockers, leukotriene antagonists, and rarely TNFalpha antagonists and IL-1/1b antagonists help prevent the released mediators from acting on the targeted cell. ${ }^{[8-13]}$

In addition, our patient was found to have hematologic malignancy involvement as well. There have been reported of hematologic malignancies in patients with SM. There were five reported cases of non-Hodgkin's malignant lymphoma arising in patients with SM. ${ }^{[14]}$ However, it is still unclear whether these cases were coincidental findings or if SM or any MCAD contributes to higher risk of hematologic or solid malignancies.

The wide spectrum of MCAS presentation continues to make its recognition challenging to researchers as well as clinicians. Diagnosing MCAS is challenging due to the episodic nature of symptoms, the multisystem involvement, and difficulty in obtaining timely labs during an episode. The clinical heterogeneity in presentation as seen with our patient who presented with predominant psychiatric complaints contributes to the challenge in recognizing MCAS. However, in patients with chronic symptoms of flushing, pruritus, headache, and gastrointestinal symptoms, MCA should be suspected and workup (see Figure 3). Patients with MCAS have poor quality of life and often feel significantly better on therapy. Continued efforts in developing effective method to help clinicians recognize MCAS will be the cornerstone in diagnosing and treating MCAS.

\section{CONFLICTS OF INTEREST DisClosure}

The authors have declared no conflicts of interest.

\section{REFERENCES}

[1] Carter MC, Metcalfe DD, Komarow HD. Mastocytosis. Immunol Allergy Clin North Am. 2014 Feb; 34(1): 181-96. PMid:24262698 https://doi.org/10.1016/j.iac.2013.09.001

[2] Bischoff SC. Role of mast cells in allergic and non-allergic immune responses: comparison of human and murine data. Nature Reviews Immunology. 2007 February; 7: 93-104. PMid:17259966 https://doi.org/10.1038/nri2018

[3] Unna P. Beitrage zur Anatomie und Pathogenese der urticarial simplex und pigmentosa. Monatschrift der praktiskchen dermatologie. 1887; 6: 9-18.

[4] Ellis JM. Urticaria pigmentosa. Arch. Pathology. 1949; 48(5): 42635 .

[5] Efrati P, Klajman A, Spitz H. Mast cell leukemia? Malignant mastocytosis with leukemia-like manifestations. Blood. 1957 Oct; 12(10): 869-82. PMid:13471655

[6] Molderings GJ, Brettner S, Homann J, et al. Mast cell activation disease: a concise practical guide for diagnostic workup and therapeutic options. J. Hematol. Oncol. 2011; 4: 10-7. PMid:21418662 https://doi.org/10.1186/1756-8722-4-10

[7] Sonneck K, Florian S, Müllauer L, et al. Diagnostic and subdiagnostic accumulation of mast cells in the bone marrow of patients with anaphylaxis: Monoclonal mast cell activation syndrome. Int Arch Allergy Immunol. 2007; 142(2): 158-64. PMid:17057414 https://doi.org/10.1159/000096442
[8] Valent P, Akin C, Escribano L, et al. Standards and standardization in mastocytosis: Consensus statement on diagnostics, treatment recommendations and response criteria. Eur J Clin Invest. 2007; 37: 435-53. PMid:17537151 https://doi.org/10.1111/j.1365-2362.20 $07.01807 . x$

[9] Valent P, Akin C, Arock M, et al. Definitions, criteria and global classification of mast cell disorders with special reference to mast cell activation syndromes: A consensus proposal. Int Arch Allergy \& Immunol. 2012; 157(3): 215-25.

[10] Afrin LB. Presentation, Diagnosis, and Management of Mast Cell Activation Syndrome. Nova Science. 2013: 155-232.

[11] Metcalfe DD. Mast cell and mastocytosis. Blood. 2008; 12: 946-56. PMid:18684881 https://doi.org/10.1182/blood-2 007-11-078097

[12] Valent P, Horny HP, Escribano L, et al. Diagnostic criteria and classification of mastocytosis: A consensus proposal. Leuk Res. 2001, 25: 603-25. https://doi.org/10.1016/S0145-2126(01) 000 38-8

[13] Afrin LB, Butterfield JH, Raithel M, et al. Often seen, rarely recognized: mast cell activation disease-a guide to diagnosis and therapeutic options. Ann Med. 2016; 48(3): 190. PMid:27012973 https://doi.org/10.3109/07853890.2016.1161231

[14] King PH, Hutchinson RM. Blood findings in generalized mastocytosis: Coexistent non-Hodgkin's lymphoma. Br J Haematol. 1991 Mar; 77(3): 436. PMid:2012771 https://doi.org/10.1111/j.1365 $-2141.1991 . t b 08597 . x$ 